\title{
Accuracy enhancement in the estimation of molecular hydration free energies by implementing the intramolecular hydrogen bond effects
}

\author{
Kee-Choo Chung and Hwangseo Park
}

\begin{abstract}
Background: The formation of intramolecular hydrogen bonds (IHBs) may induce the remarkable changes in molecular physicochemical properties. Within the framework of the extended solvent-contact model, we investigate the effect of implementing the IHB interactions on the accuracy in estimating the molecular hydration free energies.

Results: The performances of hydration free energy functions including and excluding the IHB parameters are compared using the molecules distributed for SAMPL4 blind prediction challenge and those in Free Solvation Database (FSD). The calculated hydration free energies with IHB effects are found to be in considerably better agreement with the experimental data than those without them. For example, the root mean square error of the estimation decreases from 2.56 to 1.66 and from 1.73 to $1.54 \mathrm{kcal} / \mathrm{mol}$ for SAMPL4 and FSD molecules, respectively, due to the extension of atomic parameter space to cope with $1 \mathrm{HBs}$.
\end{abstract}

Conclusions: These improvements are made possible by reducing the overestimation of attractive interactions between water and the solute molecules involving IHBs. The modified hydration free energy function is thus anticipated to be useful for estimating the desolvation cost for various organic molecules.

Keywords: Hydration free energy, Solvent-contact model, Genetic algorithm, Intramolecular hydrogen bond

\section{Background}

Because most biochemical processes take place in aqueous environment, their kinetic and thermodynamic aspects vary with the structural and energetic features of solute-water interactions. Hydration free energy $\left(\Delta G_{h y d}\right)$ refers to the free energy change for the transfer of a solute molecule in the gas phase to liquid water, and serves as a fundamental quantity to measure the biological activity of organic molecules. For example, $\Delta G_{h y d}$ has been useful for elucidating the strength of protein-ligand association and the efficacy of a drug molecule at the site of action [1-3]. Therefore, the precise estimation of molecular hydration free energy would have the effect of

*Correspondence: hspark@sejong.ac.kr

Department of Bioscience and Biotechnology, Sejong University, 209

Neungdong-ro, Kwangjin-gu, Seoul 143-747, Republic of Korea accelerating the pace of drug discovery. The necessity for an accurate computational method for $\Delta G_{\text {hyd }}$ prediction has become more urgent because the experimental measurements of $\Delta G_{h y d}$ lagged behind a rapid increase in the number of new organic compounds $[4,5]$.

Despite the difficulty in describing the complex solutewater interactions, a number of computational methods for $\Delta G_{\text {hyd }}$ prediction have been developed based on a variety of theoretical frameworks [6-15]. In 1993, Stouten et al. proposed a simple hydration free energy function constructed within the framework of solventcontact model [16], which placed an emphasis on the direct relation between $\Delta G_{\text {hyd }}$ and solvent-accessible volume around a solute atom [17]. Despite the simplicity in describing the solute-water interactions with the three atomic parameters for only six atom types $\left(\mathrm{C}, \mathrm{N}, \mathrm{O}, \mathrm{N}^{+}\right.$, $\mathrm{O}^{-}$, and $\mathrm{S}$ ), this hydration model was successfully applied

\section{Chemistry Central}

(c) 2015 Chung and Park. This article is distributed under the terms of the Creative Commons Attribution 4.0 International License (http://creativecommons.org/licenses/by/4.0/), which permits unrestricted use, distribution, and reproduction in any medium, provided you give appropriate credit to the original author(s) and the source, provide a link to the Creative Commons license, and indicate if changes were made. The Creative Commons Public Domain Dedication waiver (http://creativecommons.org/ publicdomain/zero/1.0/) applies to the data made available in this article, unless otherwise stated. 
to explain the structural properties of small proteins. In the previous studies, we improved the solvent-contact model to make it useful for estimating the $\Delta G_{h y d}$ values of diverse organic molecules by extending the atom types and atomic parameters to cope with a variety of chemical environments [18-20]. A good performance of this extended solvent-contact model was demonstrated in SAMPL4 blind prediction challenge for molecular hydration free energies [21]. In contrast to the successful prediction of the experimental $\Delta G_{\text {hyd }}$ values for the majority of organic molecules, the extended solvent-contact model showed a relatively poor performance with respect to the solutes molecules that are capable of establishing the intramolecular hydrogen bonds (IHBs). This imperfection has made it difficult for the hydration free energy function to be useful in practical applications.

Such a defect of the previous extended solvent-contact model is actually not surprising because the formation of IHBs may have a significant influence on the solutewater interactions due to the electron redistribution between the hydrogen-bond donor/acceptor groups. We aim in this study to further improve the solvent-contact model in such a way to precisely estimate the $\Delta G_{\text {hyd }}$ values of all the solute molecules including those involving IHBs. For this purpose, the atomic parameter space of the hydration free energy function is extended to reflect the effect of forming IHBs on the strength of solute-water interactions. The presence of a suitably positioned IHB in solute molecules was shown to improve the membrane permeability of a drug molecule in close relation with its solubility in aqueous solution [22]. Therefore, the modified hydration free energy function implementing the IHB effects seems to be useful for estimating the favorable drug-like properties, which further motivates this research.

\section{Computational methods}

Within the framework of the extended solvent-contact model, hydration free energy function for a solute molecule can be written in the following form.

$$
\Delta G_{h y d}=\sum_{i}^{\text {atoms }} S_{i}\left(O_{i}^{\max }-\sum_{j \neq i}^{\text {atoms }} V_{j} e^{\frac{r_{i j}^{2}}{2 \sigma^{2}}}\right)
$$

Here, gaussian-type envelope function with respect to the interatomic distance between solute atoms $\left(r_{i j}\right)$ and a constant $(\sigma)$ is employed to define the occupied volume to which the approach of water molecules is restricted. $S_{i}, O_{i}^{\max }$, and $V_{j}$ represent the atomic hydration energy per unit volume, the maximum atomic occupancy, and the atomic fragmental volume, respectively. The determination of these three parameters for each atom type is prerequisite for the calculation of $\Delta G_{h y d}$. In this study, we optimized the $S_{i}, O_{i}^{\max }$, and $V_{j}$ parameters by means of a standard genetic algorithm using a variety of solute molecules for which the experimental $\Delta G_{\text {hyd }}$ values were available. The organic molecules contained in Free Solvation Database (FSD) [23] and those distributed in SAMPL4 blind prediction challenge [24] were used for validating the accuracy of the optimized hydration free energy function.

\section{Preparation of training and test sets}

A total of 643 organic molecules in the latest version of FSD were divided into 439 and 200 molecules to construct the training and test sets, respectively, after excluding the four molecules (ammonia, hydrogen oxide, methylsulfinylmethane, and endosulfan alpha) that included the unique atom types unavailable in the other molecules. With respect to the separation of 639 FSD molecules into a training set and a test set, the similar molecules sharing more than $70 \%$ of atom types were collected into the same structural cluster. For a cluster containing $n$ elements, one-third of the molecules were randomly selected as the elements of the test set. If the number of molecules was less than 6 in a structural cluster, we selected only a single molecule as the element of test set to avoid the irrelevant optimization of atomic parameters. Both training and test sets were then confirmed for the inclusion of all the atom types present in FSD. To further investigate the impact of implementing the IHB effects on the accuracy of hydration free energy function, we also used 47 molecules distributed in SAMPL4 blind prediction challenge as the test set along with a training set prepared with 77 organic molecules [21]. All structures of the molecules in training and test sets are presented in Additional file 1.

Whereas the chemical diversity of SAMPL4 dataset is very limited because it includes a small number of molecules distributed as the targets for blind test, FSD contains structurally diverse molecules including more than 40 functional groups. Molecular weight, dipole moment, and experimental hydration free energy range from 16.04 to $498.66 \mathrm{~g} / \mathrm{mol}$, from 0 to 7.14 Debye, and from -25.47 to $3.43 \mathrm{kcal} / \mathrm{mol}$, respectively. These wide ranges of structure and physicochemical properties support the reasonableness of selecting FSD to validate the hydration free energy function.

3-D structures of all the solute molecules required for calculating the $\Delta G_{\text {hyd }}$ values were obtained through the quantum chemical geometry optimizations at B3LYP/6$31 G^{*}$ level with polarized continuum model for solvation. The optimized atomic coordinates were then inspected for the presence of IHB, which was defined as the nonbond interaction between polar atom and hydrogen with 
the interatomic distance shorter than $2.5 \AA$. Figure 1 shows all the molecules involving IHBs contained in the two training and test sets.

\section{Definition of atom types}

The definition of atom types is critically important in this study because they should reflect all the chemical circumstances each atom in the solute molecules can face. Because the redundant definition of atom types may cause the overfitting during the parametrizations, it is necessary to define the optimal number of atom types to warrant a good performance of the hydration free energy function. In case of SAMPL4 molecules, two additional atom types were required to describe the oxygen and hydrogen atoms involved in IHBs in addition to the existing 34 atom types defined according to the element, hybridization state, chemical bond, and number of substituents. A total of 36 atoms types were therefore needed to describe all the molecules in SAMPL4 data set. The number of atom types increased to 52 to cope with FSD molecules to represent a variety of chemical circumstances in 639 molecules.<smiles>O=C(c1ccccc1)C(O)c1ccccc1</smiles>

1<smiles>O=C1c2ccccc2C(=O)c2ccccc21</smiles>

2<smiles>O=C(O)c1ccccc1O</smiles>

3<smiles>Cc1cccc(Nc2ccccc2C(=O)O)c1C</smiles>

4<smiles>CCC(C)c1cc([N+](=O)[O-])cc([N+](=O)[O-])c1O</smiles>

9<smiles>O=c1[nH]c(=O)[nH]c(=O)[nH]1</smiles>

13<smiles>Nc1ccc(N)c2c1C(=O)c1ccccc1C2=O</smiles>

14<smiles>Nc1ccc(O)c2c1C(=O)c1ccccc1C2=O</smiles>

18<smiles>O=Cc1ccccc1O</smiles>

15<smiles>CCN(CC)c1c([N+](=O)[O-])cc(C(F)(F)F)c(N)c1[N+](=O)[O-]</smiles>

19<smiles>CCNc1nc(NC(C)C)nc(SC)n1</smiles>

16<smiles>O=C(O)c1cc(-c2ccc(F)cc2F)ccc1O</smiles>

20<smiles>Nc1ccccc1Cl</smiles>

17

Fig. 1 Structures of the solute molecules capable of forming the intramolecular hydrogen bonds. Functional groups involved in the hydrogen bonds are indicated in red. 1-3, 4-6, 7-13, and $\mathbf{1 4 - 2 1}$ belong to training set for SAMPL4, test set of SAMPL4, training set of FSD, and test set of FSD molecules, respectively. 2-Hydroxybenzaldehyde (5 and $\mathbf{1 5})$ and 1-amino-4-hydroxyanthraquinone $(\mathbf{6}$ and $\mathbf{1 8})$ are shown in duplicate because they belong to different data sets and their hydration free energies were calculated with different atomic parameters 


\section{Optimization of atomic parameters}

Three key atomic parameters should be determined to calculate the $\Delta G_{\text {hyd }}$ values using Eq. (1). Among them, $V_{i}$ values were fitted separately because they revealed a bad convergent behavior in the simultaneous optimization with $S_{i}$ and $O_{i}^{\max }$ parameters. A standard genetic algorithm was employed in the optimization of $V_{i}$ parameters as detailed in the previous papers $[19,20]$. This parameterization could be carried out successfully by minimizing the sum of differences between the van der Waals volume of a solute molecule and the sum of its all atomic $V_{i}$ parameters. $V_{i}$ parameters differ from $S_{i}$ and $O_{i}^{\max }$ in that they have wide variations among even the same atom types. This exceptional flexibility was assumed in the context that the partial volume of each atom in molecules can vary substantially with the change of the molecular structure irrespective of the atom types.

After the calculation of $V_{i}$ values for all the atoms in solute molecules, $S_{i}$ and $O_{i}^{\max }$ parameters were optimized simultaneously based on the genetic algorithm using the 3-D structures and the experimental $\Delta G_{h y d}$ values of the molecules in the training sets. This began with the construction of a generation consisting of 100 vectors whose elements were $S_{i}$ and $O_{i}^{\max }$ parameters for all possible atom types. In the second step, the half of 100 vectors was made empty with a bias toward preserving the best fit with the minimum error. These empty vectors were then filled with the new elements prepared from those of top 50. We generated the 50 new vectors in two steps. First, all $S_{i}$ and $O_{i}^{\max }$ values in the filled vectors were altered with probability 0.01 to make the transiently new vectors. The elements of these temporary vectors were then exchanged by cross breeding with probability 0.6 to replace some $S_{i}$ and $O_{i}^{\max }$ values with those in another vector. The 50 new vectors constructed in this way were finally scored together with the previous top 50 to select the new top 50. This procedure was iterated until the convergence criterion was satisfied. To score the vectors containing $S_{i}$ and $O_{i}^{\max }$ parameters as the elements, we used the error hypersurface $\left(F_{s}\right)$ given by summing the discrepancies between the experimental $\left(\Delta G_{\text {exp }}^{i}\right)$ and calculated molecular hydration free energies $\left(\Delta G_{\text {calc }}^{i}\right)$. This fitness function can be expressed as follows.

$$
F_{s}=\sum_{i}^{\text {molecules }}\left|\Delta G_{\exp }^{i}-\Delta G_{\text {calc }}^{i}\right|
$$

During the operation of genetic algorithm, the atomic parameters exhibited convergent behavior after about 2000 iterations.

\section{Results and discussion}

The hydration free energy function was optimized and validated using the two data sets. One contains 639 FSD molecules that were divided into 439 and 200 to constitute the training and test sets, respectively, and the other consists of 77 reference molecules (training set) and 47 SAMPL4 molecules (test set). Prior to the optimization of atomic parameters, we defined a total of 52 and 36 atom types to represent a variety of chemical circumstances in FSD and SAMPL4 molecules, respectively. Some abnormal atom types were required for coping with FSD molecules such as hexavalent sulfur (S.12) and pentavalent phosphorus (P.10) atoms. O-H type IHBs were found both in FSD and in SAMPL4 molecules while F-H and $\mathrm{Cl}-\mathrm{H}$ forms were present in the former only. These IHBs were identified by the conformational searches for the presence of non-bond interactions between hydrogen and polar heavy atoms with the interatomic distance shorter than $2.5 \AA$.

Table 1 lists the optimized $O_{i}^{\max }$ and $S_{i}$ values for 52 and 36 atom types defined to represent all the molecules in FSD and SAMPL4 data sets, respectively. Despite the large structural and populational differences in the constituent molecules, the $O_{i}^{\max }$ and $S_{i}$ values optimized with 439 FSD molecules compare reasonably well with those obtained using 77 molecules to represent 47 SAMPL4 molecules. The squared linear correlation coefficients $\left(R^{2}\right)$ to compare the parametrizations with the two training sets amount to 0.79 and 0.83 for $O_{i}^{\max }$ and $S_{i}$ values, respectively. The atomic $V_{i}$ parameters are omitted in Table 1 because they were allowed to vary in accordance with the position in molecules even in the case of the same atom types. In the strict sense, each atom in all the molecules may have its own unique $V_{i}$ value.

Despite the structural diversity of the molecules in the training sets, the optimized atomic parameters have a tendency consistent with general atomic properties. We note in this regard that the $O_{i}^{\max }$ values appear to get larger with the increase in atomic radius from hydrogen to the second- and third-period elements. Oxygen and fluorine atoms have the lower $O_{i}^{\max }$ values than carbon and nitrogen, which is consistent with the smaller atomic radii of the former than the latter. Thus, we can obtain the physically reasonable $O_{i}^{\max }$ values through the definitions of 52 and 36 atoms types for FSD and SAMPL4 molecules, respectively.

In contrast to the relative similarities among the $O_{i}^{\max }$ values for varying atom types, the $S_{i}$ parameters appear to undergo a large change with the variation of atom types even in the case of the same element. Nonetheless, the optimized $S_{i}$ values also exhibit a trend that can be elucidated with the electronic structures of individual atoms. For example, various carbon atoms have positive or very 
Table 1 The optimized maximum atomic occupancy $\left(O_{i}^{\max }\right)$ and atomic solvation parameters $\left(S_{i}\right)$ for all the atom types defined for FSD and SAMPL4 molecules

\begin{tabular}{|c|c|c|c|c|c|}
\hline \multirow[t]{2}{*}{ Atom type } & \multirow[t]{2}{*}{ Description } & \multicolumn{2}{|c|}{$O_{i}^{\max }\left(\AA^{3}\right)$} & \multicolumn{2}{|c|}{$S_{i}\left(\mathrm{kcal} / \mathrm{mol}^{3}\right)$} \\
\hline & & FSD & SAMPL4 & FSD & SAMPL4 \\
\hline C.3_1 & $\mathrm{sp}^{3}$ carbon with 1 substituent & 396.8 & 350.8 & 0.429 & 1.619 \\
\hline C.3_2 & $\mathrm{sp}^{3}$ carbon with 2 substituents & 372.4 & 368.3 & 0.524 & 0.143 \\
\hline C.3_3 & $\mathrm{sp}^{3}$ carbon with 3 substituents & 361.9 & 382.5 & -0.429 & 0.095 \\
\hline C.3_4 & $\mathrm{sp}^{3}$ carbon with 4 substituents & 379.4 & 377.0 & 1.222 & 0.794 \\
\hline C.2_1 & $\mathrm{sp}^{2}$ carbon with 1 substituent & 360.3 & 339.5 & 2.048 & 0.905 \\
\hline C.2_2 & $\mathrm{sp}^{2}$ carbon with 2 substituents & 365.1 & 354.8 & -0.905 & 0.873 \\
\hline C.2_3 & $\mathrm{sp}^{2}$ carbon with 3 substituents & 391.3 & 353.8 & -1.222 & -0.540 \\
\hline C.1_1 & sp carbon with 1 substituent & 377.0 & NA & -0.905 & NA \\
\hline C.1_2 & sp carbon with 2 substituents & 351.6 & NA & 0.143 & NA \\
\hline C.ar_2 & Aromatic carbon with 2 substituents & 392.9 & 381.1 & -1.000 & -0.889 \\
\hline C.ar_3 & Aromatic carbon with 3 substituents & 375.4 & 353.2 & -0.048 & 0.524 \\
\hline C.CO_1 & Carbonyl carbon with 1 substituent & 337.1 & 354.0 & -3.968 & -2.619 \\
\hline C.CO_2 & Carbonyl carbon with 2 substituents & 393.3 & 369.0 & -6.444 & -1.746 \\
\hline N.1_1 & sp nitrogen with 1 substituent & 404.0 & NA & -10.079 & NA \\
\hline N.2_2 & $\mathrm{sp}^{2}$ nitrogen with 2 substituents & 424.4 & NA & -11.556 & NA \\
\hline N.3_1 & $\mathrm{sp}^{3}$ nitrogen with 1 substituent & 351.6 & 384.9 & -9.333 & -10.318 \\
\hline N.3_2 & $\mathrm{sp}^{3}$ nitrogen with 2 substituents & 437.6 & 364.4 & -10.238 & -10.333 \\
\hline N.3_3 & $\mathrm{sp}^{3}$ nitrogen with 3 substituents & 454.6 & 393.7 & -14.921 & -12.302 \\
\hline N.ar & Aromatic nitrogen & 357.8 & 352.4 & -8.222 & -11.349 \\
\hline N.pl_1 & Planar nitrogen with 1 substituent & 396.8 & 358.9 & -10.159 & -12.460 \\
\hline N.pl_2 & Planar nitrogen with 2 substituents & 330.0 & 367.5 & -10.873 & -11.667 \\
\hline N.pl_3 & Planar nitrogen with 3 substituents & 358.7 & 408.9 & -8.444 & -11.905 \\
\hline N.am_1 & Amide nitrogen with 1 substituent & 398.9 & NA & -8.429 & NA \\
\hline N.am_2 & Amide nitrogen with 2 substituents & 391.1 & NA & -9.603 & NA \\
\hline N.am_3 & Amide nitrogen with 3 substituents & 399.2 & NA & -3.635 & NA \\
\hline N.no2 & Nitrogen in nitro group & 357.9 & 372.2 & -4.444 & -4.921 \\
\hline $0.3 \_1$ & $\mathrm{sp}^{3}$ oxygen with 1 substituent & 330.8 & 366.2 & -13.556 & -11.619 \\
\hline $0.3 \_2$ & $\mathrm{sp}^{3}$ oxygen with 2 substituents & 304.4 & 311.4 & -5.714 & -5.873 \\
\hline O.pl_1 & Planar oxygen with 1 substituent & NA & 316.2 & NA & -10.619 \\
\hline O.pl_2 & Planar oxygen with 2 substituents & NA & 346.8 & NA & -6.825 \\
\hline O.es_1 & $\mathrm{sp}^{3}$ oxygen in carboxylic acids & 309.5 & 327.8 & -6.508 & -8.413 \\
\hline O.es_2 & $\mathrm{sp}^{3}$ oxygen in esters & 319.8 & 333.3 & 1.778 & -2.603 \\
\hline 0.2 & $\mathrm{sp}^{2}$ oxygen & 302.4 & 347.6 & -7.619 & -9.683 \\
\hline O.no2 & Oxygen in nitro group & 342.1 & 338.9 & -0.476 & 0.825 \\
\hline O.intra & Oxygen involved in intramolecular hydrogen bond & 323.0 & 309.0 & -1.270 & -3.810 \\
\hline S.12 & Sulfur with 12 valence electrons & 410.3 & NA & -3.810 & NA \\
\hline S.3_1 & $\mathrm{sp}^{3}$ sulfur with 1 substituent & 429.4 & NA & -0.762 & NA \\
\hline S.3_2 & $\mathrm{sp}^{3}$ sulfur with 2 substituents & 402.4 & NA & -6.857 & NA \\
\hline 5.2 & $\mathrm{sp}^{2}$ sulfur & 428.6 & NA & 1.556 & NA \\
\hline S.pl & Planar sulfur & 409.5 & NA & -0.190 & NA \\
\hline $\mathrm{F}$ & Fluorine & 284.1 & NA & -3.714 & NA \\
\hline F.intra & Fluorine involved in intramolecular hydrogen bond & 277.9 & NA & 1.365 & NA \\
\hline $\mathrm{Cl}$ & Chlorine & 452.4 & 408.7 & -0.794 & -3.016 \\
\hline Clintra & Chlorine involved in intramolecular hydrogen bond & 458.1 & NA & -0.317 & NA \\
\hline $\mathrm{Br}$ & Bromine & 500.8 & NA & -1.778 & NA \\
\hline । & lodine & 549.2 & NA & -1.556 & NA \\
\hline P.10 & Phosphorus with 10 valence electrons & 404.4 & NA & -4.095 & NA \\
\hline
\end{tabular}


Table 1 continued

\begin{tabular}{|c|c|c|c|c|c|}
\hline \multirow[t]{2}{*}{ Atom type } & \multirow[t]{2}{*}{ Description } & \multicolumn{2}{|c|}{$O_{i}^{\max }\left(\AA^{3}\right)$} & \multicolumn{2}{|c|}{$S_{i}\left(\mathrm{kcal} / \mathrm{mol}^{3}\right)$} \\
\hline & & FSD & SAMPL4 & FSD & SAMPL4 \\
\hline H.C & Hydrogen bonded to carbon & 201.6 & 182.2 & 0.111 & -0.444 \\
\hline H.N3 & Hydrogen bonded to $\mathrm{sp}^{3}$ nitrogen & 254.4 & 212.7 & -5.556 & -2.540 \\
\hline H.Np & Hydrogen bonded to planar nitrogen & 207.9 & 223.8 & -1.746 & -1.159 \\
\hline $\mathrm{H} . \mathrm{O} 3$ & Hydrogen bonded to $\mathrm{sp}^{3}$ oxygen & 236.7 & 204.8 & -7.159 & -9.286 \\
\hline H.Op & Hydrogen bonded to planar oxygen & NA & 237.3 & NA & -10.889 \\
\hline H.Oa & Hydrogen bonded to carboxylic acid group & 230.2 & 202.4 & -3.444 & -6.190 \\
\hline H.S & Hydrogen bonded to sulfur & 228.9 & NA & -5.397 & NA \\
\hline H.intra & Hydrogen involved in intramolecular hydrogen bond & 222.2 & 210.0 & -3.190 & -4.222 \\
\hline
\end{tabular}

low negative $S_{i}$ values in both parametrizations with FSD and SAMPL4 molecules, which indicates their insignificant interactions with water. This is consistent with the low solubility of hydrocarbons in water. However, the decrease of the average $S_{i}$ values in the order of $s p^{3}, s p^{2}$, and $s p$ carbons indicates that the interaction of a solute carbon atom with water becomes more favorable due to the increase of the s-character in the hybridization state of atomic orbitals. Such a dependence of $S_{i}$ on the extent of s-character may be elucidated in the context that the increased s-character in the hybrid atomic orbitals has the effect of increasing the electronegativity, which would culminate in facilitating the intermolecular dipole-dipole interactions with water. Besides the increased electronegativity, the decrease in the number of substituents on the carbon with high s-character would also have the effect of lowering the $S_{i}$ value because water molecules can approach the central carbon readily along a line perpendicular to the molecular plane. Due to the combined effects of the increased polarity and the increased water accessibility, both atom types of carbonyl carbons (C.CO_1 and C.CO_2) have very negative $S_{i}$ values. This is physically acceptable in terms of the high solubility of carbonyl compounds in water.

Consistent with the critical roles of nitrogen and oxygen atoms in the stabilization of organic molecules in water, their optimized $S_{i}$ values are highly negative for most atom types. This may be invoked to explain the long-range attractive electrostatic interactions with bulk water and to the capability to form the local hydrogen bonds with water molecules, both of which contribute to making the solute-water interactions thermodynamically favorable. However, the $S_{i}$ values of oxygens appear to become less negative in the presence of IHBs in solute molecules. The optimized $S_{i}$ parameters of the oxygen atoms involved in IHBs (O.intra) amount to -1.270 and -3.810 in the parametrizations for FSD and SAMPL molecules, respectively, as compared to the corresponding average $S_{i}$ values of -5.349 and -6.851 for the rest of oxygens. This can be related with the partial loss of electron density on the oxygen due to the electron transfer from its non-bond orbital to the antibonding $\sigma^{*}$ orbital of hydrogen-bond donor group, which is characteristic of a normal hydrogen bond.

The $S_{i}$ values of the hydrogen atoms bonded to heteroatoms are much more negative than those of hydrocarbons (Table 1). This is consistent with the accumulation of positive charges due to the electron withdrawal by the neighboring heteroatoms that are more electronegative than carbon. In accordance with the increase of $S_{i}$ value for O.intra, however, the H.intra atoms also reveal the less negative $S_{i}$ values than the other hydrogens adjacent to the heteroatoms. For instance, the $S_{i}$ parameter of H.intra converges to -3.190 and -4.222 in the optimization with FSD and SAMPL4 molecules, respectively, in comparison to the corresponding average values of -4.660 and -6.013 for the other hydrogens attached to the electronegative heteroatoms. This can be elucidated also in the context of the electron transfer from the hydrogen-bond acceptor atom and the resulting partial neutralization of the positive charges on H.intra atoms.

Figure 2 shows the linear correlation diagrams between the experimental hydration free energies and those calculated with the optimized hydration free energy function with respect to the training and the test set comprising 77 reference molecules and 47 SAMPL4 molecules, respectively. To examine the effect of parameterizing IHBs in solute molecules on the accuracy of hydration free energy function, we compare the results of $\Delta G_{\text {hyd }}$ prediction with the atomic parameters for IHBs to those without them. With respect to the test set consisting of 47 SAMPL4 molecules, we obtain the $R^{2}$ value of 0.849 in the absence of IHB parameters (Fig. 2b). However, the $R^{2}$ value of the fitting for the test set increases to 0.913 due to the reflection of IHB effects in the parametrization (Fig. 2d). When the positions of the solute molecules involving IHB in 

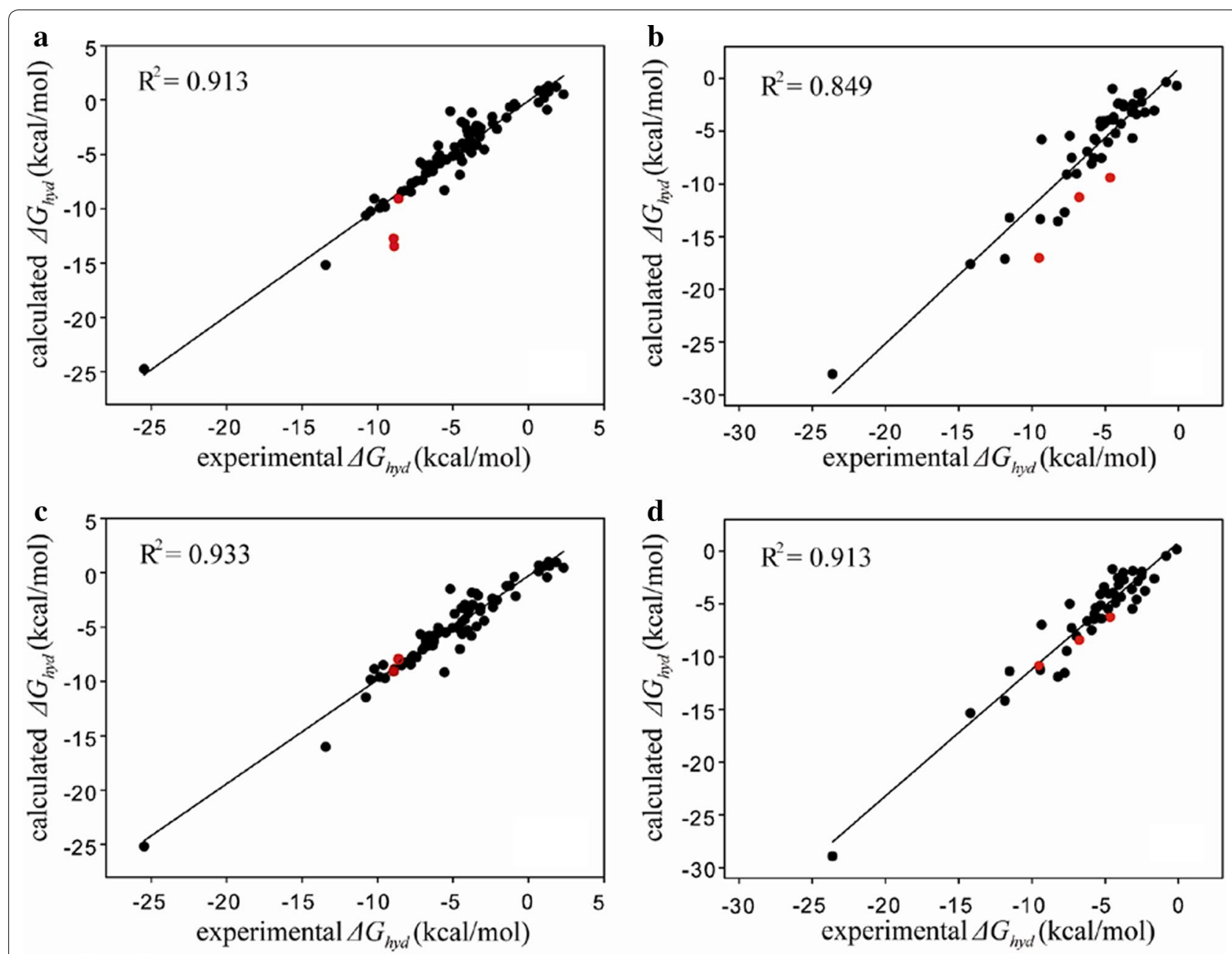

Fig. 2 Linear correlation diagrams for the experimental versus calculated hydration free energies for a training set of 77 molecules without IHB parameters, $\mathbf{b}$ test set of 47 SAMPL4 molecules without IHB parameters, c training set of 77 molecules with IHB parameters, and $\mathbf{d}$ test set of 47 SAMPL4 molecules with IHB parameters. Indicated in red circles are the solute molecules involving IHBS

the fitting (red circles in Fig. 2) are compared, it follows immediately that the major contribution to the enhancement of the correlation comes from the better estimation of their $\Delta G_{\text {hyd }}$ values. It is also noteworthy that the extension of atomic parameter space to cope with IHBs leads to the decrease in the difference between the $R^{2}$ values of the training and test sets from 0.064 to 0.020 , which indicates the reduced possibility of overtraining during the operation of genetic algorithm. Furthermore, the root mean square error (RMSE) for estimating the $\Delta G_{\text {hyd }}$ values of SAMPL4 molecules appears to decrease substantially from 2.56 to $1.66 \mathrm{kcal} / \mathrm{mol}$ due to the additional parameterization for IHBs. These results exemplify the necessity of separate atomic parameters for IHBs in solute molecules to improve the accuracy in estimating the molecular hydration free energies.
The importance of implementing the IHB effects in estimating the $\Delta G_{h y d}$ values is further demonstrated in the validation results for FSD molecules. Figure 3 illustrates the correlations between the $\Delta G_{\text {hyd }}$ values of FSD molecules measured from experiments and those calculated with Eq. (1) and the optimized atomic parameters. It is a common feature in the fittings with SAMPL4 and FSD data sets that the improvement of $R^{2}$ value due to the augmentation of the atomic parameters is even more significant in the test set than in the training set. We obtain the $R^{2}$ value of 0.825 without the IHB parameters for the test set comprising 200 molecules (Fig. 3b), as compared to 0.903 for the training set of 439 molecules. This large difference in the $R^{2}$ values implies that the atomic parameters should be over-trained in the absence of the atom types for IHB. The $R^{2}$ value of the 

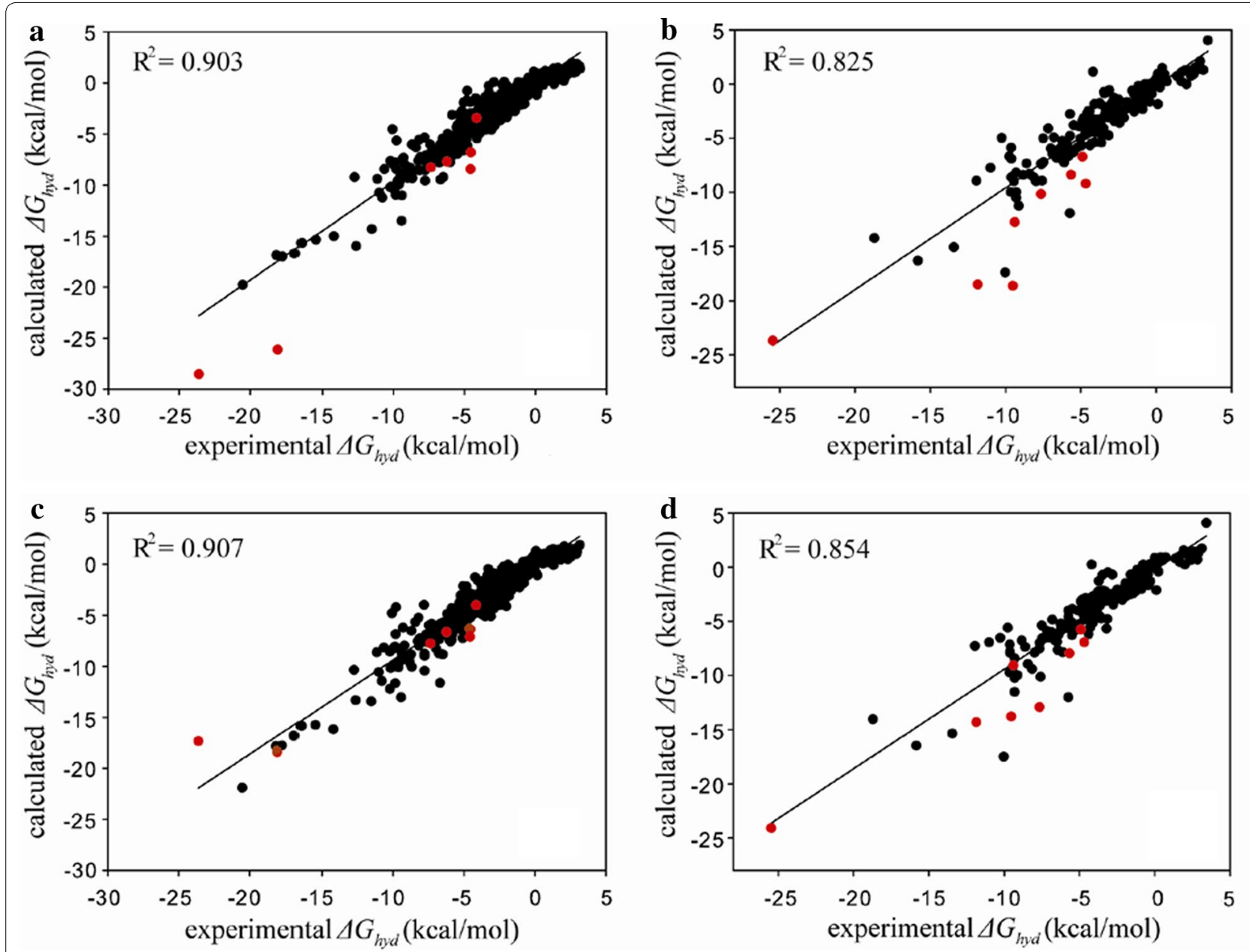

Fig. 3 Linear correlation diagrams for the experimental versus calculated hydration free energies for a training set of 439 FSD molecules without IHB parameters, $\mathbf{b}$ test set of 200 FSD molecules without IHB parameters, c training set of 439 FSD molecules with IHB parameters, and $\mathbf{d}$ test set of 200 FSD molecules with IHB parameters. Indicated in red circles are the solute molecules involving IHBS

test set appears to increase to 0.854 in the fitting for the $\Delta G_{\text {hyd }}$ results obtained under consideration of IHB effects (Fig. 3d). This significant predictability enhancement can be attributed in a large part to the better prediction of the $\Delta G_{\text {hyd }}$ values of the solute molecules with IHBs, which can be inferred from their positional shifts in the linear correlation diagrams (red circles in Fig. 3). Due to the additional parameterization for IHBs, RMSE for the predicted $\Delta G_{\text {hyd }}$ values of test set molecules amounts to only $1.54 \mathrm{kcal} / \mathrm{mol}$, as compared to $1.73 \mathrm{kcal} / \mathrm{mol}$ in the hydration model excluding the IHB effects. The significant enhancements in $R^{2}$ and RMSE values confirm the necessity for extending the atomic parameter space to cope with IHBs for the better estimation of molecular hydration free energies.

As can be inferred from the decrease of the $R^{2}$ value from 0.913 (Fig. 2d) to 0.854 (Fig. 3d), our extended solvent-contact model exhibits a worse performance for FSD molecules than for SAMPL4 ones in terms of the correlation with the experimental data. This may be attributed to the requirement of much more atom types for FSD than for SAMPL4 molecules because chemical environments are more diverse in the former than in the latter. Furthermore, we find that some atom types for sulfur, $s p$ carbon, and $s p^{2}$ nitrogen atoms are rarely observed in FSD data set, which makes it difficult for the corresponding atomic parameters to be fully optimized due to the insufficient number of representatives in the training set.

The performance of hydration free energy function was further evaluated using the new training and test sets constructed by merging those for SAMPL4 and FSD datasets, the results of which are summarized in Fig. 4. The $R^{2}$ value of 0.814 is obtained for the test set 

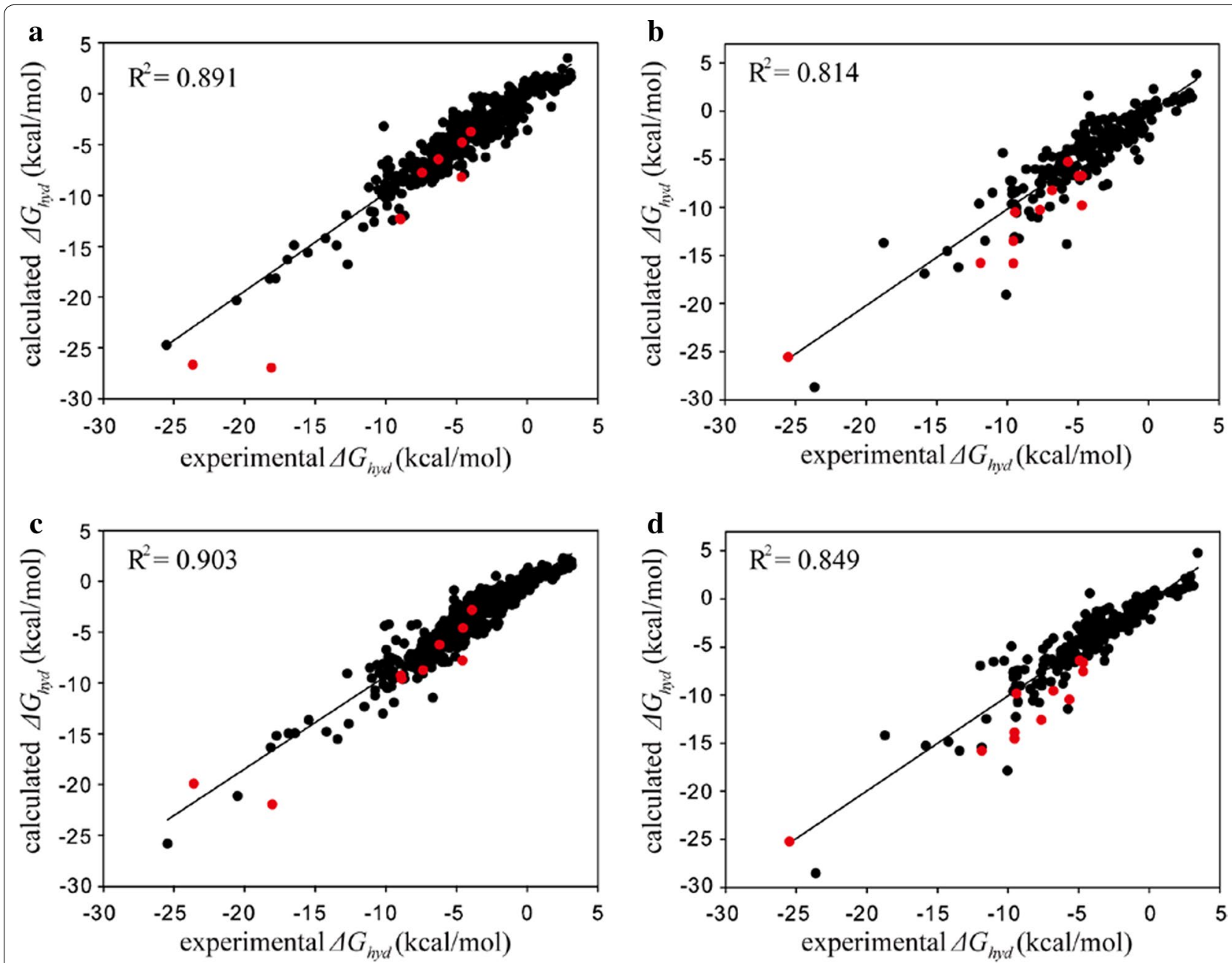

Fig. 4 Linear correlation diagrams for the experimental versus calculated hydration free energies for a training set of a total of 516 molecules without IHB parameters, $\mathbf{b}$ test set of 247 SAMPL4 and FSD molecules without IHB parameters, $\mathbf{c}$ training set of 516 molecules with IHB parameters, and d test set of 247 SAMPL4 and FSD molecules with IHB parameters. Indicated in red circles are the solute molecules involving IHBS

comprising 47 SAMPL4 plus 200 FSD molecules in the absence of the IHB parameters (Fig. 4b), which is even smaller than that $(0.891)$ for the training set comprising a total of 516 (77 plus 439) molecules (Fig. 4a). Judging from such a large difference in the $R^{2}$ values, the atomic parameters seem to be over-trained in the absence of the IHB parameters. The $R^{2}$ value of the test set increases to 0.849 in the fitting for the experimental and computational $\Delta G_{\text {hyd }}$ values if the IHB effects are reflected in the parametrizations (Fig. 4d). Furthermore, the augmentation of the IHB atomic parameters leads to the decrease of RMSE for $\Delta G_{\text {hyd }}$ predictions of the new test set molecules from 1.94 to $1.68 \mathrm{kcal} / \mathrm{mol}$. The validation results obtained with the merged dataset are thus consistent with those for SAMPL4 and FSD datasets in the context that $R^{2}$ and RMSE values increase and decrease, respectively, due to the implementation of IHB effects. This consistency confirms that the extension of atomic parameter space is necessary to enhance the accuracy in estimating the $\Delta G_{\text {hyd }}$ values of the solute molecules involving IHBs.

Compared in Table 2 are the experimental and computational $\Delta G_{\text {hyd }}$ values of the solute molecules (1-21 shown in Fig. 1) that involve IHBs. A high discrepancy between experimental and computational results is observed for most IHB molecules that belong to the test sets (4-6 and 14-21) if the IHB effects are neglected. The average unsigned error (AUE) of the calculated $\Delta G_{\text {hyd }}$ values for these solute molecules amounts to $4.12 \mathrm{kcal} / \mathrm{mol}$, which is much higher than that for all the molecules included in the two test sets $(1.28 \mathrm{kcal} / \mathrm{mol})$. The most inaccurate result is obtained with the deviation 
Table 2 Experimental $\left(\Delta G_{\text {hyd,exp }}\right)$ and calculated $\left(\Delta G_{\text {hyd,calc }}\right)$ hydration free energies (in $\mathrm{kcal} / \mathrm{mol}$ ) of 21 molecules capable of establishing the intramolecular hydrogen bonds

\begin{tabular}{|c|c|c|c|c|c|}
\hline \multirow[t]{2}{*}{ Compound } & \multirow[t]{2}{*}{$\Delta G_{\text {hyd,exp }}$} & \multicolumn{2}{|c|}{ No IHB parameter } & \multicolumn{2}{|c|}{$\begin{array}{l}\text { IHB parameters } \\
\text { included }\end{array}$} \\
\hline & & $\Delta G_{\text {hyd,calc }}$ & Error & $\Delta G_{\text {hyd,calc }}$ & Error \\
\hline 1 & -8.62 & -9.05 & 0.43 & -7.97 & 0.65 \\
\hline 2 & -8.90 & -13.46 & 4.56 & -8.86 & 0.04 \\
\hline 3 & -8.95 & -12.74 & 3.79 & -9.08 & 0.13 \\
\hline 4 & -6.78 & -11.28 & 4.50 & -8.43 & 1.65 \\
\hline 5 & -4.68 & -9.41 & 4.73 & -6.25 & 1.57 \\
\hline 6 & -9.53 & -17.03 & 7.50 & -10.87 & 1.34 \\
\hline 7 & -4.16 & -3.41 & 0.75 & -4.01 & 0.15 \\
\hline 8 & -4.55 & -6.80 & 2.25 & -6.38 & 1.83 \\
\hline 9 & -6.23 & -7.71 & 1.48 & -6.65 & 0.42 \\
\hline 10 & -23.62 & -28.50 & 4.88 & -17.32 & 6.30 \\
\hline 11 & -4.58 & -8.43 & 3.85 & -7.12 & 2.54 \\
\hline 12 & -7.37 & -8.25 & 0.88 & -7.76 & 0.39 \\
\hline 13 & -18.06 & -26.12 & 8.06 & -18.40 & 0.34 \\
\hline 14 & -11.85 & -18.50 & 6.65 & -14.30 & 2.45 \\
\hline 15 & -4.68 & -9.18 & 4.50 & -6.92 & 2.24 \\
\hline 16 & -7.65 & -10.14 & 2.49 & -12.90 & 5.25 \\
\hline 17 & -4.91 & -6.71 & 1.80 & -5.73 & 0.82 \\
\hline 18 & -9.53 & -18.64 & 9.11 & -13.79 & 4.26 \\
\hline 19 & -5.66 & -8.36 & 2.70 & -7.95 & 2.29 \\
\hline 20 & -9.40 & -12.74 & 3.34 & -9.06 & 0.34 \\
\hline 21 & -25.47 & -23.69 & 1.78 & -24.08 & 1.39 \\
\hline
\end{tabular}

1-3, 4-6, 7-13, and 14-21 belong to training set for SAMPL4, test set of SAMPL4, training set of FSD, and test set of FSD molecules, respectively

of $9.11 \mathrm{kcal} / \mathrm{mol}$ for $\mathbf{1 8}$ in which multiple IHBs are established.

It is a common feature in the $\Delta G_{\text {hyd }}$ values of most IHB molecules calculated without the IHB parameters that they are underestimated substantially when compared to the corresponding experimental results. This indicates that the large errors in the calculated $\Delta G_{\text {hyd }}$ values of IHB molecules stem from the overestimation of attractive solute-water interactions. However, the implementation of IHB parameters leads to a dramatic decrease in the discrepancies between the experimental and calculated $\Delta G_{\text {hyd }}$ values of the solute molecules capable of forming IHBs. For example, the AUE value for $\mathbf{4}-\mathbf{6}$ and 14-21 decreases to only $2.01 \mathrm{kcal} / \mathrm{mol}$ due to the additional parameterizations for IHB, which is relatively similar to that for all the solute molecules in the two test sets $(1.09 \mathrm{kcal} / \mathrm{mol})$. Thus, the accuracy enhancement in the present extended-solvent contact model can be attributed to the alleviation of the overestimation of the attractive solute-water interactions.

Related with the substantial contribution of IHBs to molecular hydration free energy, it needs to be noted that the experimental $\Delta G_{\text {hyd }}$ value increases from -11.85 in 14 to $-9.53 \mathrm{kcal} / \mathrm{mol}$ in 18 in response to the replacement of $-\mathrm{NH}_{2}$ with $-\mathrm{OH}$ moiety. This is quite unexpected because the $\Delta G_{\text {hyd }}$ value of aniline $(-5.49 \mathrm{kcal} /$ $\mathrm{mol})$ is higher than that of phenol $(-6.61 \mathrm{kcal} / \mathrm{mol})$. In this regard, we obtain a slightly higher $\Delta G_{h y d}$ value for $\mathbf{1 4}$ than for $\mathbf{1 8}$ in the absence of IHB parameters, which is more consistent with the experimental results for aniline and phenol than those for $\mathbf{1 4}$ and 18. On the other hand, the $\Delta G_{\text {hyd }}$ value of $\mathbf{1 4}$ becomes more negative than that of $\mathbf{1 8}$ if they are calculated with the hydration free energy function implementing the IHB parameters. It can thus be argued that the relative strength of solute-water interactions for IHB molecules may be predicted incorrectly in the absence of IHB parameters. The governing role of IHB in the hydration behaviors of solute molecules was also observed in the experimental measurements of dielectric relaxation [25].

The higher $\Delta G_{h y d}$ value of $\mathbf{1 8}$ than 14 can be understood in terms of the difference in the strength of IHB. Because phenolic group is more acidic than anilinic one, the former should form the stronger hydrogen bond with the vicinal carbonyl oxygen than the latter. Therefore, a substantial amount of electron density seems to be transferred from the non-bonding orbital of the carbonyl oxygen to the anti-bonding $\sigma^{*}$ molecular orbital of phenolic $\mathrm{O}-\mathrm{H}$ bond, which has the effect of lowering the polarities of both chemical moieties involved in IHB. This culminates in the weakening of long-range attractive electrostatic interactions with bulk solvent as well as in reducing the possibility of forming local hydrogen bonds with water molecules. In this viewpoint, the problem of overestimating the attractive solute-water interactions seems to be inevitable unless the IHB effects are taken into account in the hydration free energy function.

It should be noted that some atom types such as S.pl and F.intra (Table 1) are rare in the training set. The low occurrence of certain atom types in the training set may affect the accuracy of hydration free energy function. For example, the differences between the experimental and calculated hydration free energies of $\mathbf{1 6}$ and $\mathbf{1 9}$ amount to 69 and $40 \%$, respectively (Table 2). These large deviations can be attributed to the incomplete optimization of atomic parameters due to the low occurrence of S.pl and F.intra in the training set.

Actually, the accuracy in estimating the $\Delta G_{\text {hyd }}$ values can be enhanced by increasing the number of atom types in such a way to cope with all the solute atoms in different chemical environments. For example, it would be desirable to distinguish the carbonyl carbons from the normal $\mathrm{sp}^{2}$ carbons to reflect the significant positive atomic charge developed due to the adjacent carbonyl oxygen. Some additional atoms types for nitrogen and 
oxygen seem to be required as well for drug-like molecules because they include a variety of heterocyclic moieties. However, the subdivision of atom types may have a negative effect on the accuracy when the experimental data for training are insufficient for optimizing the parameters associated with the newly created atom types. For example, the low occurrences of S.pl and F.intra atoms in the training set lead to a large deviation of the calculated $\Delta G_{\text {hyd }}$ values of $\mathbf{1 6}$ and $\mathbf{1 9}$ from the experimental ones (Table 2) due to the incomplete optimization of atomic parameters. To maximize the accuracy in $\Delta G_{\text {hyd }}$ predictions, therefore, the extension of atom types should be limited to the cases for which the atomic parameters can be fully optimized with the corresponding experimental data.

The merit of the present extended solvent-contact model lies in the capability to elucidate the unusual possibility that the substitution of a more polar moiety than the existing one may render the solute molecule more hydrophobic due to the formation of IHBs. In this regard, some peptidomimetic molecules proved to become more hydrophobic with the substitution of two polar groups to establish an IHB, which led to the enhancement of membrane permeability without impairing the other druglike properties [22]. The hydration free energy function implementing the IHB parameters is therefore anticipated to be useful for screening drug candidates with good membrane permeability.

However, the present hydration model seems to be a little imperfect as a useful $\Delta G_{\text {hyd }}$ estimator because the errors for some IHB molecules amount to more than $4 \mathrm{kcal} / \mathrm{mol}$ (Table 2). With respect to this large deviation, we note that the experimental $\Delta G_{h y d}$ data are available only for a small number of IHB molecules in publicly accessible chemical databases. It is therefore difficult to fully optimize the IHB parameters due to the rarity of reference data. We expect that the performance of our extended solvent-contact model will be further enhanced considerably in the future in the presence of abundant experimental $\Delta G_{\text {hyd }}$ data for IHB molecules.

Despite the difficulty in collecting the reference data, the hydration free energy function appears to be improved to a significant extent due to the implementation of atomic IHB parameters as illustrated in Figs. 2 and 3. This improvement is made possible because the risk of over-fitting due to the increased atomic parameters can be surmounted effectively by reducing the overestimation of attractive interactions between water and IHB molecules. The RMSE values of 1.66 and $1.54 \mathrm{kcal} /$ mol associated with $\Delta G_{\text {hyd }}$ prediction for SAMPL4 and FSD molecules, respectively, seem to be insignificant because the experimental $\Delta G_{\text {hyd }}$ data cover a wide range of $\sim 30 \mathrm{kcal} / \mathrm{mol}$. The accuracy of the present extended solvent-contact model is comparable in terms of $R^{2}$ value to those of some high-level quantum chemical calculations and statistical simulations with all-atom models [26-29]. The characteristic feature that discriminates our hydration model from the others lies in that one can compute the $\Delta G_{h y d}$ values in a straightforward way from the potential energy function without significant computational burden.

Although it is shown in this study that $\Delta G_{\text {hyd }}$ values can be estimated effectively with the extended solvent-contact model implementing the IHB effects, there remains the possibility of further improvement. For example, the entropic contribution to $\Delta G_{\text {hyd }}$ needs to be calculated separately because the hydration free energy function in Eq. (1) lacks the entropic term. Although the determination of hydration entropy $\left(\Delta S_{\text {hyd }}\right)$ had been considered a very difficult task, it was reported recently that $\Delta S_{\text {hyd }}$ could be estimated with reasonable accuracy by means of combining free energy perturbation (FEP) method and scaled particle theory (SPT) to calculate the electrostatic and hydrophobic contributions of solute-water interactions separately [30]. Therefore, the combination of $\Delta H_{\text {hyd }}$ and $\Delta S_{\text {hyd }}$ values calculated respectively with our extended solvent-contact model and the hybrid SPT/FEP approach seems to serve as a useful method for estimating the $\Delta G_{\text {hyd }}$ values of small molecules.

\section{Conclusions}

The formation of IHBs in solute molecules may lead to the weakening of solute-water interactions due to the charge transfer between the hydrogen-bond acceptor/ donor groups. This would have the effect of reducing the polarity of solute molecules, and cause the unexpected increase in the $\Delta G_{\text {hyd }}$ values. In this study, we examined the effect of implementing the IHB interactions on the accuracy of the extended solvent-contact model for $\Delta G_{\text {hyd }}$ prediction using SAMPL4 and FSD molecules. As a consequence of augmenting the atomic parameters for IHBs, the calculated $\Delta G_{h y d}$ values became in better agreement with experimental data. For example, the $R^{2}$ values between the experimental and calculated $\Delta G_{\text {hyd }}$ values increased from 0.849 to 0.913 and from 0.825 to 0.854 for SAMPL4 and FSD molecules, respectively, due to the extension of atomic parameters to cope with IHBs. Furthermore, the RMSE values of the estimation decreased from 2.56 to $1.66 \mathrm{kcal} / \mathrm{mol}$ and from 1.73 to $1.54 \mathrm{kcal} /$ mol for SAMPL4 and FSD molecules, respectively, in the presence of the atomic IHB parameters. The comparisons of the calculated $\Delta G_{\text {hyd }}$ values indicated that such a significant accuracy enhancement stemmed from the reduction of the overestimation for the attractive electrostatic interactions between water and IHB molecules. This was in turn made possible by properly describing the electron 
redistribution between IHB acceptor and donor groups, which has the effect of weakening their polarities. Thus, the results in this study exemplified the necessity for the augmentation of atomic parameters according to the specific chemical environments to improve the accuracy of the hydration free energy function.

\section{Additional file}

Additional file 1. Contains chemical structures, experimental and calculated solvation free energies of 763 molecules used in this study.

\section{Authors' contributions}

KCC: Performed all calculations and summarized the results, HP: Developed idea and wrote paper. Both authors read and approved the final manuscript.

\section{Acknowledgements}

This work was supported by Basic Science Research Program through the National Research Foundation of Korea (NRF) funded by the Ministry of Education, Science and Technology (NRF-2011-0022858).

\section{Competing interests}

The authors declare that they have no competing interests.

Received: 4 August 2015 Accepted: 12 November 2015 Published online: 25 November 2015

\section{References}

1. Zou X, Sun Y, Kuntz ID (1999) Inclusion of solvation in ligand binding free energy calculations using generalized-Born model. J Am Chem Soc 121:8033-8043

2. Lindström $A$, Edvinsson $L$, Johansson $A$, Andersson $C D$, Andersson IE, Raubacher F, Linusson A (2011) Postprocessing of docked protein-ligand complexes using implicit solvation models. J Chem Inf Model 51:267-282

3. Lipinski CA, Lombardo F, Dominy BW, Feeney PJ (1997) Experimental and computational approaches to estimate solubility and permeability in drug discovery and development settings. Adv Drug Deliv Rev 23:3-25

4. Corbett PT, Leclaire J, Vial L, West KR, Wietor JL, Sanders JKM, Otto S (2006) Dynamic combinatorial chemistry. Chem Rev 106:3652-3711

5. Cougnon FBL, Sanders JKM (2012) Evolution of dynamic combinatorial chemistry. Acc Chem Res 45:2211-2221

6. Pitera JW, van Gunsteren WF (2001) One-step perturbation methods for solvation free energies of polar solutes. J Phys Chem B 105:11264-11274

7. Marenich AV, Cramer CJ, Truhlar DG (2008) Perspective on foundations of solvation modeling: the electrostatic contribution to the free energy of solvation. J Chem Theory Comput 4:877-887

8. Nicholls A, Mobley DL, Guthrie JP, Chodera JD, Bayly Cl, Cooper MD, Pande VS (2008) Predicting small-molecule solvation free energies: an informal blind test for computational chemistry. J Med Chem 51:769-779

9. Mobley DL, Bayly Cl, Cooper MD, Shirts MR, Dill KA (2009) Small molecule hydration free energies in explicit solvent: an extensive test of fixedcharge atomistic simulations. J Chem Theory Comput 5:350-358

10. Shivakumar D, Williams J, Wu Y, Damm W, Shelley J, Sherman W (2010) Prediction of absolute solvation free energies using molecular dynamics free energy perturbation and the OPLS force field. J Chem Theory Comput 6:1509-1519

11. Boyer RD, Bryan RL (2012) Fast estimation of solvation free energies for diverse chemical species. J Phys Chem B 116:3772-3779

12. Sergiievskyi VP, Fedorov MV (2012) 3DRISM multi-grid algorithm for fast solvation free energy calculations. J Chem Theory Comput 8:2062-2070
13. Sumi T, Mitsutake A, Maruyama Y (2015) A solvation-free-energy functional: a reference-modified density functional formulation. J Comput Chem 36:1359-1369

14. Abella JR, Cheng SY, Wang Q, Yang W, Ren P (2014) Hydration free energy from orthogonal space random walk and polarizable force field. J Chem Theory Comput 10:2792-2801

15. Klamt A, Diedenhofen M (2015) Calculation of solvation free energies with DCOSMO-RS. J Phys Chem A 119:5439-5445

16. Stouten PFW, Frömmel C, Nakamura H, Sander C (1993) An effective solvation term based on atomic occupancies for use in protein simulations. Mol Simul 10:97-120

17. Colonna-Cesari F, Sander C (1990) Excluded volume approximation to protein- solvent interaction. The solvent contact model. Biophys $J$ 57:1103-1107

18. Kang H, Choi H, Park H (2007) Prediction of molecular solvation free energy based on the optimization of atomic solvation parameters with genetic algorithm. J Chem Inf Model 47:509-514

19. Choi H, Kang H, Park H (2013) Extended solvent-contact model for protein solvation: test cases for dipeptides. J Mol Graphics Model 42:50-59

20. Choi H, Kang H, Park H (2013) New solvation free energy function comprising intermolecular solvation and intramolecular self-solvation terms. J Cheminform 5:8

21. Park H (2014) Extended solvent-contact model approach to SAMPL4 blind prediction challenge for hydration free energies. J Comput Aided Mol Des 28:175-186

22. Rafi SB, Hearn BR, Vedantham P, Jacobson MP, Renslo AR (2012) Predicting and improving the membrane permeability of peptidic small molecules. J Med Chem 55:3163-3169

23. Mobley DL, Guthrie JP (2014) FreeSolv: a database of experimental and calculated hydration free energies, with input files. J Comput Aided Mol Des 28:711-720

24. Mobley DL, Wymer KL, Lim NM, Guthrie JP (2014) Blind prediction of solvation free energies from the SAMPL4 challenge. J Comput Aided Mol Des 28:135-150

25. Shikata T, Okuzono M (2013) Hydration/dehydration behavior of polyalcoholic compounds governed by development of intramolecular hydrogen bonds. J Phys Chem B 117:2782-2788

26. Want SC, Beal PA, Tantillo DJ (2010) Covalent hydration energies for purine analogs by quantum chemical methods. J Comput Chem 31:721-725

27. Sampson C, Fox T, Tautermann CS, Woods C (2015) Skylaris: a "Steppng Stone" approach for obtaining quantum free energies of hydration. J Phys Chem B 119:7030-7040

28. Huggins DJ, Payne MC (2013) Assessing the accuracy of inhomogeneous fluid solvation theory in predicting hydration free energies of simple solutes. J Phys Chem B 117:8232-8244

29. Modesto Orozco M, Colominas C, Luque FJ (1996) Theoretical determination of the solvation free energy in water and chloroform of the nucleic acid bases. Chem Phys 209:19-29

30. Choi H, Kang H, Park H (2015) Computational prediction of molecular hydration entropy with hybrid scaled particle theory and free-energy perturbation method. J Chem Theory Comput 11:4933-4942

\section{Publish with ChemistryCentral and every} scientist can read your work free of charge

"Open access provides opportunities to our colleagues in other parts of the globe, by allowing anyone to view the content free of charge."

W. Jeffery Hurst, The Hershey Company.

- available free of charge to the entire scientific community

- peer reviewed and published immediately upon acceptance

- cited in PubMed and archived on PubMed Central

- yours - you keep the copyright

Submit your manuscript here:

http://www.chemistrycentral.com/manuscript/ 